\title{
Toward a semantic approach for automatic composition of Learning Grid Services
}

\author{
Gustavo Gutiérrez-Carreón ${ }^{1}$, Thanasis Daradoumis ${ }^{1}$ and Josep Jorba ${ }^{1}$ \\ ${ }^{1}$ Open University of Catalonia, Av. Tibidabo 39-43 - 08035 Barcelona, Spain \\ \{ggutierrezc, adaradoumis, jjorbae\}@uoc.edu
}

\begin{abstract}
Due to their potential advantages, the use of Grid technologies toward the development of collaborative and personalized learning frameworks has been considerably increased. These learning frameworks can be constructed based on the distributed learning services and resources available in a learning Grid environment. A problem still unsolved is how to use and integrate low-level learning services to compose more complex high-level services or tools that can be useful to both tutors and learners. In that sense, on the one hand, semantic description of Grid learning services appears to be a powerful tool that can be used to discover suitable learning services depending on the system's semantic capabilities. On the other hand, it can be employed to carry out a matching process among the learning services located in a learning framework to obtain the best fit according to specific functional parameters. These parameters represent significant characteristics of a learning Grid environment. This paper presents an initial effort that integrates schema and ontology matching methods that aim to develop a model to cope with the complex problem of automatic composition of Grid based learning tools and their portals.
\end{abstract}

\section{Introduction}

Distance e-learning emerges as one of promising means for people to learn online. Although there is a substantial increase in computer and network performance in recent years, mainly as a result of faster hardware and more sophisticated software, there are still problems in the fields of integrating various resources towards enabling distance e-learning.

In the field of Grid services, an important issue is how to achieve the correct integration of interorganizational and heterogeneous services on the Web. If no single Grid service can satisfy the functionality required by the user, there should be a possibility to combine existing services together in order to fulfill the request. In that sense a lot of efforts have been made to develop techniques and methods for search [1,2], discovery [3] , matching [4] and composition $[5,6,7,8,9]$ of grid and web services using semantic description, which showed that important advantages could be achieved if compared with syntactic search.

This paper, on the one hand, provides a review of some related work that takes advantage of grid technologies to build up collaborative and personalized e-learning frameworks. On the other hand, it takes the above research work into account and proposes an initial model for the automatic composition of Grid based learning services based on the semantic capabilities and metadata of e-learning frameworks. The rest of the paper is organized as follows: In section 2 we review some research work related to the use of Grid technologies in e-learning frameworks and some other related to the use of semantic description to develop searching and composition models. Then, Section 3 goes further and describes a conceptual model that explores a new way to enhance the automatic composition of Grid Learning Tools and Services based on technologies for web services discovery and matching, whereby Section 4 illustrates how the model is applied in a collaborative learning portal. Section 5 concludes the paper and describes future work.

\section{Collaborative and personalized learning frameworks based on Grid.}

Service-based educational systems open new ways in the usability of the Grid as their primary requirements include the provision of adequate services for sharing, syndicating heterogeneous resources and relevant content discovery. The Learning Grid paradigm aims at making use of the collective intelligence and the personalized use of a range of available and potential Grid Learning services. In that sense, Grid Learning Services, taking advantage of 
technological support of Web services in general and Grid services in particular, have fundamentally changed the way that e-learning frameworks were developed.

In [10] the authors describe a platform of e-learning based on Grid service technologies. In this platform the supply of virtual learning services designated for students, instructors and course suppliers is based on the resource administration for group collaboration based on Grid, allowing ubiquous access to information and taking advantage of the potentiality of the computer systems. On the one hand, the advantage of this proposal is that it is the first one that elaborates on the use of Grid resources and their description through Grid technologies, in particular WSDL[11]. On the other hand, it dictates the need for the development of a semantic model description that enables a more complete description of learning resources.

A further work proposes an Agent-Based Robust Collaborative Virtual Environment for E-Learning in the Service Grid [12]. In this virtual environment, all Web resources and services are accessed via service encapsulation, which may result in a more scalable and robust collaborative learning architecture. A very remarkable aspect of this work is the way it uses to implement complex services from more basic ones, though no use of semantic description is made to allow the automatic composition of complex services from lower level ones.

KGCL, a Knowledge-Grid-Based Cooperative Learning Environment [13], supports the cooperation between a person and the computer at a knowledge level, and allows the enrichment not only of the resources in the Knowledge Grid but also of the users' knowledge by means of knowledge refinement, knowledge reuse and the online meeting of participants. The KGCL prototype has been currently applied and is available for online use. Experiments have shown that the environment can promote the effectiveness of group work. This system has also shown the great impact that Grid technologies can have even though no model of semantic description was implemented that could improve its performance.

\subsection{Semantic Description of Grid Learning Services}

There is some research work related to the semantic description of Grid Learning Services. OntoEdu [14] is a flexible platform for online learning which is based on diverse technologies like ubiquous computing, ontology engineering, Web semantics and computational Grid. It is compound of five parts: user adaptation, automatic composition, educative ontologies, a module of services and a module of contents; among these parts the educative ontology is the main one. The main objectives of OntoEdu are to obtain reusability of concepts, adaptability for users and devices, automatic composition, as well as scalability in functionality and performance. In the near future, this platform aims to be adapted to a Grid environment so that it can carry out its activities based on distributed computing.

The work developed in [6] presents a workflow framework for pervasive learning objects composition by employing a Grid services flow language. The learning objects are distributed in heterogeneous environments which have been used to allow effective collaboration and the reuse of learning objects; this fact can help users learn with no limitations of time and space. This work shows the great opportunities that exist in those research groups which make use of Grid technology to develop innovative, pervasive and ubiquous learning scenarios. Though this research work is still encountered at an initial phase, it can be further enhanced by the application of semantic description of learning services.

Finally in [1], the authors have constructed an ontological description for collaborative work tools that allow one to make a manual search of the diverse resources that these tools provide within a Grid environment with the minimum of technical knowledge. This work proposes a Grid-based tool, called Gridcole, which can serve as a basis to implement different conceptual approaches of Gridbased semantic description of learning services, thus extending and endowing it with an innovative, pervasive and ubiquous projection.

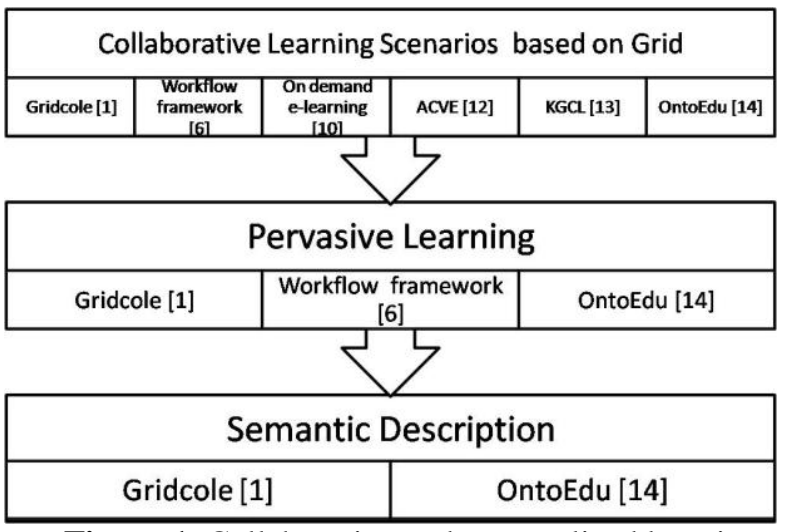

Figure 1. Collaborative and personalized learning frameworks based on Grid

In sum, the works presented above try to provide a solution to the complex problems related with collaboration, personalization and grid learning 
services semantic description (Figure 1), but they are either limited in semantic expressiveness for matching services or they do not face at all the difficult task of using and integrating low-level learning services to compose more complex ones. Both these features could greatly enhance and facilitate the tutor's and learners' labor in a complex web-based learning scenario. The complexity of composing Learning Services is related, on one hand, with the distributed nature of resources and services in a Learning Grid and, on the other hand, with the difficulty to locate services based only on syntactic information. Due to this, we need to construct mechanisms to describe semantic capabilities of learning services and to develop methods for using that information to discover and match learning services depending on our needs. This issue will be detailed in the next section.

\section{Grid Learning Services Composition}

There are three principal motivations for Learning Grid Services Composition: build a more powerful service using basic existing services, fulfill service requester's requirement better, and enhance resource reuse while reducing the cost and time of a new service development. IMS Global Learning Consortium proposes an abstract framework [15] representing a set of services used to construct an e-learning system in its broadest sense; it focuses more on the support of elearning systems and covers the possible range of eLearning architectures that could be constructed from a set of defined services. This work takes into account this Abstract Framework and the associated IMS specifications produced to realize the exchange of information between the identified services which are adopted here in a manner that is suitable for a collaborative and personalized learning framework based on Learning Grid where the resource and service are distributed into a network. Fig 2. shows the dependencies between the different "layers" of the framework.

The Learning Application composition process consists of identifying sub-tasks of the learning process, locating suitable Learning application Services to construct each process, locating suitable Common Services to construct each learning service, formatting the Learning and Common services into a service flow and executing the service flow to achieve a task which is the goal of the learning process.

The core stage is the composition of learning web services and their adaptation to the needs of a learner or group of learners. Such a composition is carried out by retrieving previously registered objects. Once composed and packaged as learning objects, these composite processes can be executed and then instantiated and adapted to the learner's particular needs.

These adaptations can be realized, either by predefined rules implemented into the process description and driven by the learner behavior, or in a supervised manner. In the later case, the instructional designer can return to the composition tools to adapt the process.

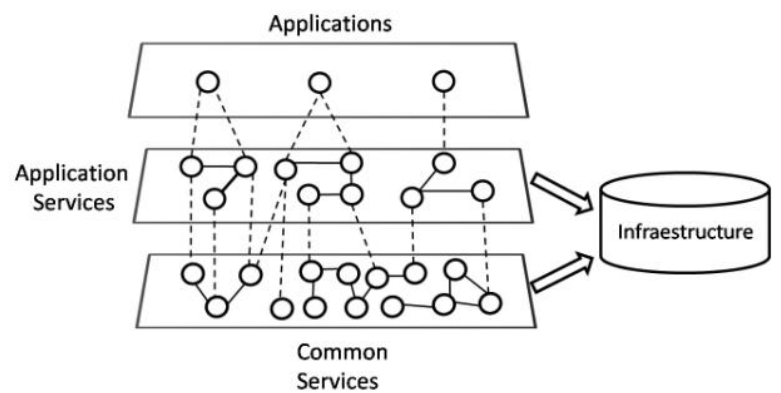

Figure 2. IMS Abstract Framework

\subsection{A Conceptual Model for Grid Learning Services automatic composition}

Let us consider a learning Grid based on Globus Tolkit 4.0 (GT4) [16] as a set of resources and services distributed in a network with the service model based on the IMS abstract framework, where learning services can be composed by others allocated in different repositories inside the network. The model we propose for the automatic composition of learning services is based on the use of the defined syntactic and semantic characteristics of the different levels of services involved in the Learning Abstract Framework. The Monitoring and Discovery Services (MDS) of GT4 are mainly concerned with the collection, distribution, indexing, archival, and otherwise processing information about the state of various resources, services, and system configurations. The information collected is used to either discover new services or resources, or to enable monitoring of system status. The Index service is the central component of the GT4 MDS implementation. The MDS also has the Trigger service, which collects information and compares that data against a set of conditions defined in a configuration file. When a condition is met an action is executed. The MDS-Index service and the MDS-Trigger service are specializations of a general Aggregator Framework. The Aggregator Framework is a software framework for building software services that collect and aggregate data. These services are also known as 
aggregator services. The design of the model is presented in Fig. 3 and is described in detail below.

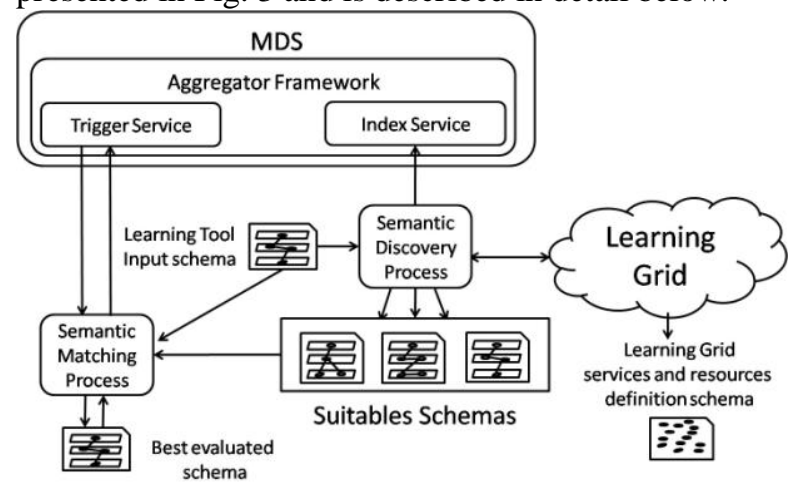

Figure 3. A conceptual Model of Grid Learning services automatic composition

\subsection{Defining a semantic schema}

Using web languages, such as RDF, DAML+OIL, and OWL, it is possible to create semantically rich data models that are denominated semantic schemas [17]. These semantic schemas are made up of triples (subject-predicate-object), where subjects and objects are entities, and predicates indicate relationships between those entities. Users can define their own properties, as well as their own classes. Instances of these classes can then be created and described with values for related properties. In these schemas there is more implicit information than it can be usually found in their text representation. Each triple forms a graph with two nodes connected by an edge. Each instance can have several properties, and that graph can be expanded to have many nodes connected to the central instance. Finally, when two instances are connected via a property, their respective sub-graphs become connected.

The proposed model claims to provide a Semantic Description for all services and resources included in the learning Grid. The first step needed to carry out the automatic composition is to generate a Semantic schema of the learning tool or learning services that will be composed. This schema can be constructed using the different tools of descriptions at the "syntactic level" through WSDL or, at the semantic level, through service ontologies included in OWL-S, WSMO, SWSF and WSDL-S [4].

\subsection{A Semantic Discovery of Grid Learning Services}

Discovery is the process of finding Web services with a given capability [18]. In GT4, the Index service interacts with data sources via standard WSRF resource property and subscription/notification interfaces. An Index service can potentially collect information from many sources and publish it in only one place. Various WSRF registrations with the Index service are maintained as Service Group Entries by the Index service. The contents of the Index service can be queried via XPath queries.

In general, a semantic discovery process relies on semantic annotations, containing high-level abstract descriptions of service requirements and behavior. Metadata is an essential element in semantic discovery with the capability to expand service descriptions with additional information. The achievement of dynamic composition and automation of services involves discovering new services at run time by software components without human interaction. SOAP provides a description of message transport mechanisms, whereas WSDL describes the interface used by each learning service. However, neither SOAP nor WSDL are of any help for the automatic location of learning services on the basis of their capabilities.

In our model, once the semantic schema of the tool or learning service that we want to build is designed, we have to pass it to our discovery process that will locate a set of different level services in the Learning Grid. The operation of these services as a whole allows us to carry out the processes defined in the schema. This process consists primarily on comparing inputs and outputs [19] of a service as semantic concepts represented in the schema to incorporate semantics about learning services accessible by a discovery service [18]. The result of the search will be a group of suitable schemas that conforms to the functional process described in our initial schema passed to the Index Service.

\subsection{A Semantic Matching of Grid Learning Services}

Schema and ontology matching aim at identifying semantic correspondences between metadata structures or models such as database schemas, XML message formats, and ontologies. Solving such match problems are of key importance to service interoperability and data integration in numerous application domains [20].

In our model, the resulting schemas of discovery process will be compared to the initial schema through a Matching process that interacts with the Trigger Service and that is based on a structural matching approach and on a taxonomy matcher and whose result will be the best evaluated schema for our learning tool or services. The taxonomy matcher draws on the given taxonomic Metadata to deduce whether two elements 
are related semantically. The result of this matching process will be a ranking of semantic matching results. This ranking can be used in conjunction with other user-defined constraints to inform of an exact, or potentially useful web-service capability match.

Comparing our conceptual model with the work presented in [1, 6, 14 and 16], our approach represents a complete alternative solution since, on the one hand, we provide a multi-level learning services composition method that enables the construction of complex learning services by means of other low level services, depending on the nature of the learning abstract framework. On the other hand, our approach takes advantage of the semantic and syntactic characteristics of learning services, which facilitates a totally automatic construction of new learning tools based on others previously created.

\section{Example Scenario using Sakai: A Leaning Collaborative portal}

We selected Sakai [21] as an initial effort to simulate our semantic supported composition model into a collaborative and personalized application, in particular to obtain significant information that can be integrated with GT4 to generate Learning Grid portals (Figure 4). Sakai is an open source online Collaboration and Learning Environment developed in Jakarta Tomcat. It has tools (applications), application services and common services repository. Many users of Sakai deploy it to support teaching and learning, ad hoc group collaboration, support for portfolios and research collaboration.

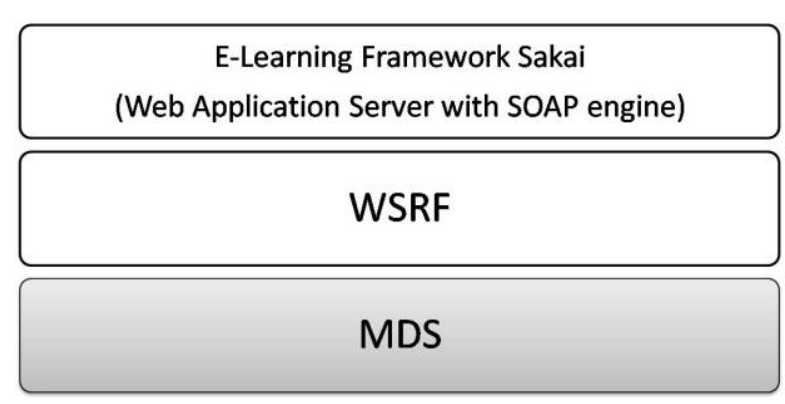

Figure 4. Integration of Sakai in a GT4 environment

The first step is to construct a semantic schema using an ontology language (in this example we use OWL-S) for tools and services contained in Sakai's repository as it is shown below (Figure 5).

Next, we turn to define the semantic schema of tool or service to be composed. In this case we want to compose a forum application. To this end we define the semantic schema of our request trying to define the most detailed information about the specific Tool or Learning Service to be composed.
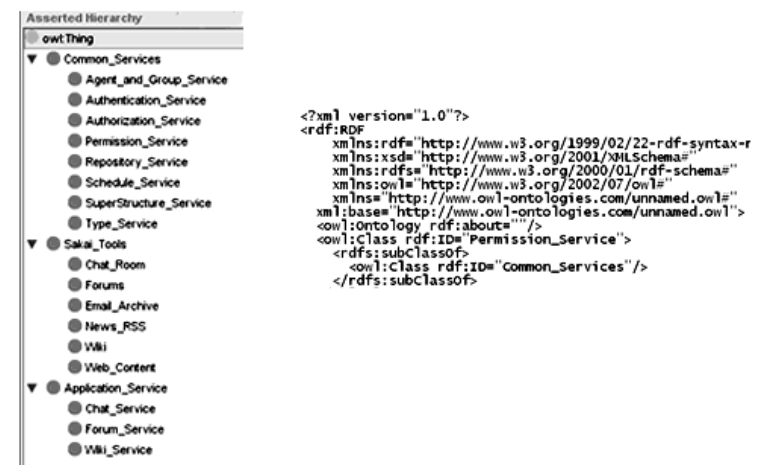

Figure 5. Semantic schema of Sakai Tools and Services

Through an initial searching process, we simulated an indexing process of a discovery service adapted to semantic searching to obtain an initial set of suitable Learning services according to the semantic definition of the forum tool. Subsequently, we went through a Matching process based on a structural matching approach and on a taxonomy matcher. The result of this process is shown in Figure 6.

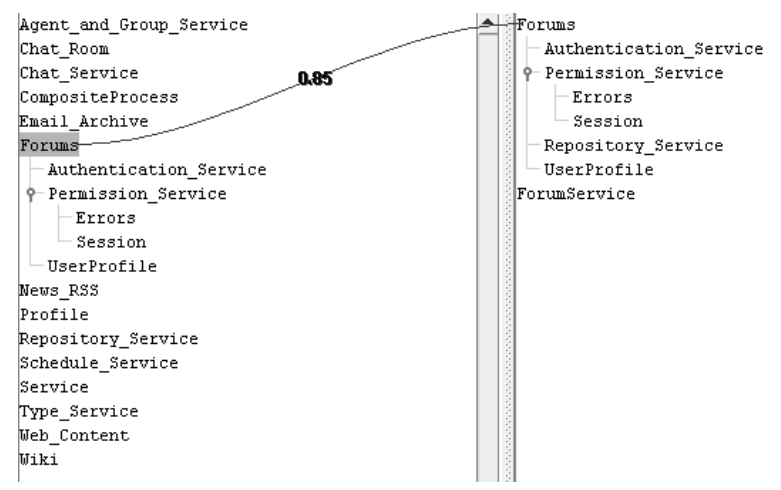

Figure 6. Best evaluated schema resulting from the matching process.

To implement this scenario we used Protegé 3.1 [22] to define the semantic schemas and COMMA ++ [20] to simulate the discovery and matching process.

\section{Conclusions and future work}

In this paper we reviewed some research work related to the use of technologies supporting Grid to develop learning frameworks that focus on 
collaborative and personalized improvements and some work that uses semantic description for the search, discovery and composition of Learning Services included in a Learning Grid. In that sense we first highlighted the importance of defining a contextually based semantic model of a learning scenario, which is particularly significant in semantically based automatic service searching, discovery, and composition. Then we made an initial effort to describe a model that can be used for automatic composition of Learning Grid Services based on schema and ontology matching. We used the IMS abstract framework layer model to structure both a semantic schema which defines distributed resources and services available in a Learning Grid and another semantic schema which defines desired learning services or tools to be composed. Both schemas are used, on the one hand, to compare inputs and outputs and discovery related learning services and, on the other hand, to realize a match process based on a structural matching approach and a taxonomy matcher. Future work aims at the full implementation of the conceptual model presented in this work in a GT4 environment with a real time composition of learning collaborative scenarios and portals based on the grid.

\section{References}

[1] Guillermo Vega-Gorgojo, Miguel L. Bote-Lorenzo, Eduardo Gómez-Sánchez, Yannis A. Dimitriadis, Juan I. Asensio-Pérez, Semantic Search of Learning Services in a Grid- Based Collaborative System, Proc. of the Fifth IEEE/ACM Int. Symposium on Cluster Computing and the Grid (CCGrid 2005), Workshop on Collaborative and Learning Applications of Grid Technology and Grid Education (CLAG) 2005, 19-26, Cardiff, UK, May 2005.

[2] Naveen Srinivasan, Massimo Paolucci, and Katia Sycara, An Efficient Algorithm for OWL-S Based Semantic Search in UDDI, Book Series Lecture Notes in Computer Science, Volume 3387, 2005

[3] Preeda Rajasekaran, John Miller, Kunal Verma, Amit Sheth, Enhancing Web Services Description and Discovery to Facilitate Composition, International Workshop on Semantic Web Services and Web Process Composition, 2004 [4] Beniamino Di Martino, An Ontology Matching Approach to Semantic Web Services Discovery, Lecture Notes in Computer Science, Volume 4331, 2006

[5] Jinghai Rao and Xiaomeng Su, A Survey of Automated Web Service Composition Methods, Lecture Notes in Computer Science, Volume 3387, 2005

[6] Ching-Jung Liao, Fang-Chuan Ou Yang, A Workflow Framework for Pervasive Learning Objects Composition by Employing Grid Services Flow Language. Proceedings of the IEEE International Conference on Advanced Learning Technologies (ICALT'04), pp: 840 - 841, 2004.

[7] Xiaofeng Du, William Song, Malcolm Munro, Service Composition in the Context of Grid, AHM2006, 2006
[8] Yang-Seung Jeon, Eun-Ha Song, Minyi Guo, Laurence T. Yang, Young-Sik Jeong, Jin-Tak Choi, Sung-Kook Han, Ontology-Based Composition of Web Services for Ubiquitous Computing, Lecture Notes in Computer Science, Volume 4331, 2006

[9] F. Casati, S. Ilnicki, and L. Jin. Adaptive and dynamic service composition in EFlow. In Proceedings of 12th International Conference on Advanced Information Systems Engineering(CAiSE), Stockholm, Sweden, Springer Verlag, 2000

[10] Zongwei Luo, Yulian Fei, and Junyuan Liang, On Demand E-Learning with Service Grid Technologies. Edutainment 2006: 60-69.

[11] W3C, Web Service Description Language Specification, http://www.w3.org/TR/wsdl

[12] Changqin Huang, Fuyin $\mathrm{Xu}$, Xianghua $\mathrm{Xu}$, and Xiaolin Zheng, Towards an Agent-Based Robust Collaborative Virtual Environment for E-Learning in the Service Grid. Lecture Notes in Computer Science, Volume 4088, 2006, [13] Hai Zhuge, Yanyan Li,Jia Bi, and To-yat Cheung, KGCL: A Knowledge-Grid-Based Cooperative Learning Environment. Lecture Notes In Computer Science; Volume 2436, 2002.

[14] Cui Guangzuo, Chen Fei, Chen Hu, Li Shufang, OntoEdu: A Case Study of Ontology-based Education Grid System for E-Learning, GCCCE2004 International conference, Hong Kong. 2004

[15] IMS Global Learning Consortium, IMS Abstract Framework: White Paper, 2003

[16] Bart Jacob, Michael Brown, Kentaro Fukui, Nihar Trivedi, Introduction to Grid Computing, ibm.com/redbooks, 2005

[17] Paul Mutton, Jennifer Golbeck, Visualization of Semantic Metadata and Ontologies, Computer Science, University of Kent at Canterbury, 2003

[18] Jennifer M. Schopf, Mike D'Arcy, Neill Miller, Laura Pearlman, Ian Foster, and Carl Kesselman, Monitoring and Discovery in a Web Services Framework: Functionality and Performance of the Globus Toolkit's MDS4, Argonne National Laboratory Tech Report ANL/MCS-P1248-0405, April 2005

[19] Massimo Paolucci, Takahiro Kawamura, Rerry R. Payne, and Katia Sycara. Semantic matching of web services capabilities, The Semantic Web - ISWC 2002: First International Semantic Web Conference, Sardinia, Italy, June 9-12, 2002

[20] Aumueller, D.; Do, H.H.; Massmann, S.; Rahm, E., Schema and ontology matching with COMA++, SIGMOD Conference, 2005

[21] Sakai Project, http://sakaiproject.org

[22]Holger Knublauch, Ray W. Fergerson, Natalya F. Noy and Mark A. Musen, The Proteg'e OWL Plugin: An Open Development Environment for Semantic Web Applications, 2004 